\title{
Ultrasound Guided Low Thoracic Erector Spinae Plane Block for Lumbar and Sacral Spine Surgery: Case Series
}

\author{
Ebru Biricik ${ }^{1}$; Feride Karacaer ${ }^{1}$ \\ 1 Department of Anesthesiology and Reanimation, Çukurova University Faculty of \\ Medicine, Adana, Turkey
}

Background and aims: Ultrasound (USG) guided Erector Spinae Plane (ESP) block is a regional anesthesia technique which can provide effective analgesia for thoracic region. (1) But recent cases showed that this technique can be apply for lower abdominal and spinal surgeries. Lumbar spine surgery can be associated with severe postoperative pain. We aimed to present case series which show effectiveness of the USG guided lower thoracic ESP block.

Methods: We report our experience with lower thoracic USG guided ESP block in six patients who underwent spinal surgery. All of the USG guided ESP blocks performed under general anesthesia. After induction of anesthesia the patients placed to prone position and skin disinfection provided with $10 \%$ povidoneiodine. The high frequency linear-array ultrasound transducer (Esaote, MyLab 30, Italy) was used for imaging. The 10th thoracic vertebra identified with counting down from 7th cervical spinous process and then USG placed in an approximately $3 \mathrm{~cm}$ lateral to the midline. The needle was inserted in plane with the ultrasound and advanced to the cranial-to-caudal direction to gently contact the transverse process. Then, local anesthetics $15 \mathrm{ml}$ bupivacaine $0.5 \%$ and $5 \mathrm{ml}$ lidocaine $2 \%$ injected.

Figure 1: Ultrasound Guided ESP Block Application

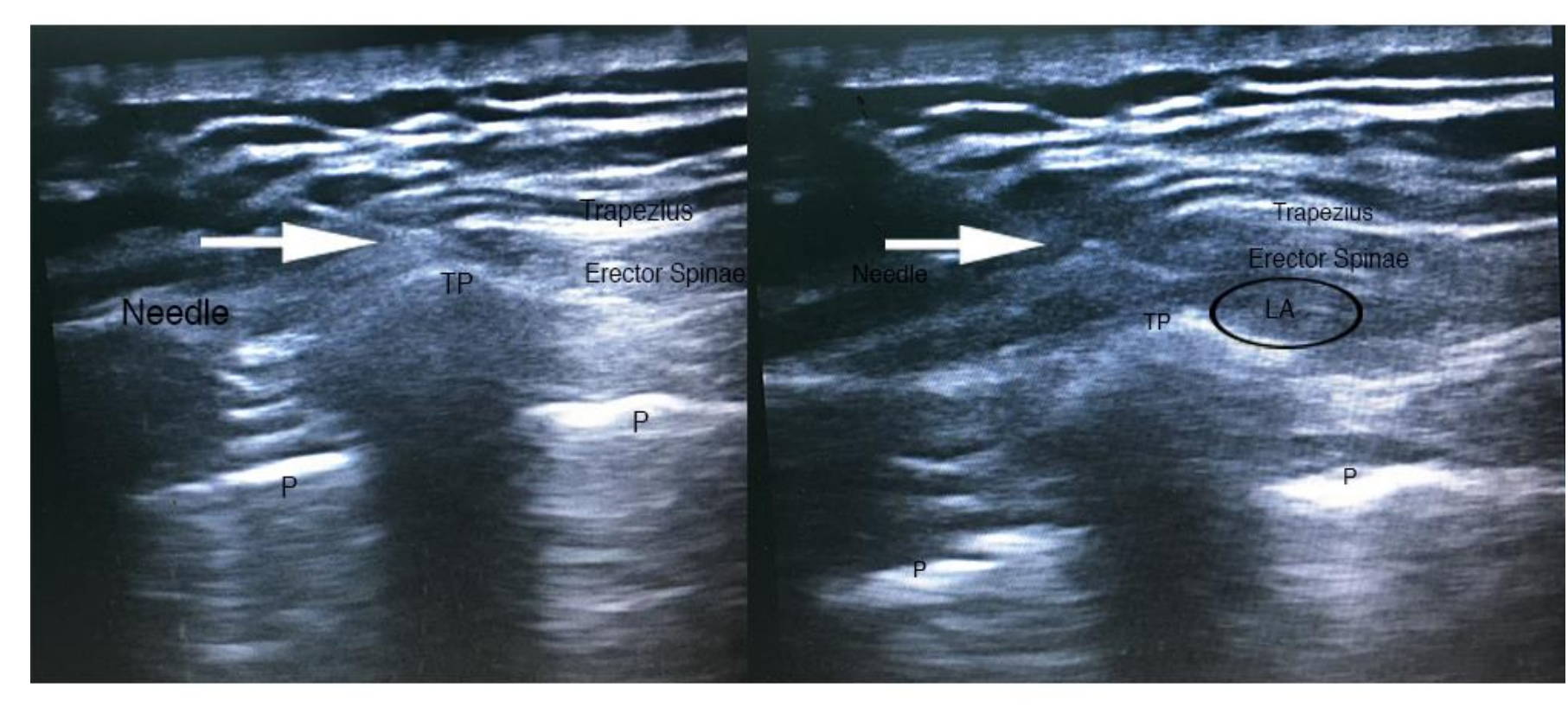

Abbreviations: P; Pleura, TP; Transvers Process, LA; Local Anesthetic

Results: Only one patient experienced 4 point of numerical rating scale at postoperative period. Demographical and surgical history of patients presented at Table 1.Five patients decelerated 3 and below NRS. We observed that ESP block has a lower risk of complication and helpful for reducing postoperative pain for lumbar and sacral spinal surgery. (Table 2) None of the patients experienced motor block.

Table 1: Demographics and Surgical History of Patients.

\begin{tabular}{|c|c|c|c|c|}
\hline $\begin{array}{c}\text { Case } \\
\text { Number }\end{array}$ & Year & Gender & Medical history & Intervention \\
\hline Case 1 & 34 & Female & $\begin{array}{c}\text { Operated } \\
\text { meningomyelocel }\end{array}$ & Lumbosacral release \\
\hline Case 2 & 64 & Female & $\begin{array}{l}\text { L3-L4 bilaterally } \\
\text { disk hernia } \\
\end{array}$ & $\begin{array}{c}\text { Bilaterally micro } \\
\text { discectomy } \\
\end{array}$ \\
\hline Case 3 & 60 & Male & $\begin{array}{l}\text { L3-L4 bilaterally } \\
\text { disk hernia }\end{array}$ & L3-L4 decompression \\
\hline Case 4 & 50 & Female & $\begin{array}{l}\text { L4-L5 bilaterally } \\
\text { disk hernia }\end{array}$ & $\begin{array}{c}\text { L4-L5 lumbar spine } \\
\text { decompression }\end{array}$ \\
\hline Case 5 & 65 & Male & $\begin{array}{l}\text { L2-3, L3-4, L4-5 } \\
\text { spinal stenosis }\end{array}$ & $\begin{array}{c}\text { L2-3, L3-4, L4-5 micro } \\
\text { discectomy and } \\
\text { foraminotomy }\end{array}$ \\
\hline Case 6 & 38 & Female & $\begin{array}{c}\text { L5-S1 spinal } \\
\text { stenosis and disk } \\
\text { hernia }\end{array}$ & $\begin{array}{l}\text { L5-S1 micro } \\
\text { discectomy and } \\
\text { foraminotomy }\end{array}$ \\
\hline
\end{tabular}

Table 2:Numerical Rating Scale Scores at Postoperative Period

\begin{tabular}{cccccccc}
\hline $\begin{array}{c}\text { Case } \\
\text { No }\end{array}$ & $\begin{array}{c}\text { NRS } \\
\text { at } \\
\text { PACU }\end{array}$ & $\begin{array}{c}\text { NRS } \\
\text { 3th }\end{array}$ & $\begin{array}{c}\text { NRS } \\
\text { 12th }\end{array}$ & $\begin{array}{c}\text { NRS } \\
\text { 24th }\end{array}$ & $\begin{array}{c}\text { Motor } \\
\text { hlockade }\end{array}$ & Analgesic & $\begin{array}{c}\text { Rescue } \\
\text { Analgesic } \\
\text { requirement }\end{array}$ \\
\hline Case 1 & 3 & 4 & 3 & 3 & None & Tramadol & Yes \\
Case 2 & 0 & 0 & 0 & 0 & None & Morphine & None \\
Case 3 & 0 & 0 & 0 & 0 & None & Morphine & None \\
Case 4 & 0 & 0 & 0 & 0 & None & Tramadol & None \\
Case 5 & 0 & 2 & 0 & 0 & None & Tramadol & None \\
Case 6 & 3 & 3 & 0 & 0 & None & Tramadol & None \\
\hline
\end{tabular}

Conclusion: We observed that low thoracic ESP block provide satisfactory postoperative analgesia after lumbar and sacral spine surgery without any complication and this technique did not cause motor block.

Reference:

1. Forero M, Adhikary SD, Lopez H, Tsui C, Chin KJ. The erector spinae plane block: a novel analgesic technique in thoracic neuropathic pain. Reg Anesth Pain Med 2016; 41: 621-7. 